\title{
Les conditions d'application de la loi du 13 juillet 1982 relative à l'indemnisation des victimes des catastrophes naturelles aux dommages dus à la sécheresse
}

\author{
How the french law (13th of july 1982) is applied \\ in compensating building damages due to drought?
}

\section{TOULEMONT}

Délégation aux Risques Majeurs

(Ministère de l'environnement) ${ }^{*}$

Rev. Franç. Géotech, n 58, pp. 27-30 (janvier 1992)

\section{Résumé}

Les dommages occasionnés aux constructions par la sécheresse ayant sévi en 1989,1990 et 1991 entrent dans le champ d'application de la loi $n^{\circ} 82-600$ du 13 juillet 1982. Ils peuvent, à ce titre, faire l'objet d'une indemnisation par les assurances, sous réserve que l'état de catastrophe soit constaté par arrêté interministériel. La prise d'un tel arrêté est fondé sur l'examen d'un dossier justificatif comportant notamment un diagnostic géotechnique. On donne ici quelques précisions sur l'établissement d'un tel diagnostic dont l'objet est de mettre en évidence la relation de causalité entre les dommages subis et l'intensité anormale du phénomène de sécheresse.

\footnotetext{
Abstract

In France, building damages due to drought are covered by compensation procedures that stem from the law of the 13th of july 1982. Compensating, paid by insurance companies, is subject to the official statement of the "state of natural disaster ". Such a statement is made as an " arrêté " signed by several ministers and based on evaluation of all supporting evidence. The file must contain a geotechnical diagnosis. This diagnosis has to show the causal ling between the "abnormal intensity of the natural agent " (as worded by the law) and the damages observed. Some details are given on how to establish a geotechnical diagnosis.
} 
1. Les années 1989 et 1990 et 1991 ont été marquées, dans la plus grande partie de notre pays, par une sécheresse que l'on peut qualifier d'exceptionnelle tant par son intensité que par sa durée. Les déficits pluviométriques records enregistrés au cours de ces trois années hydrologiques, hivers compris, associés à des températures estivales et un taux d'ensoleillement particulièrement élevés se sont traduits par un assèchement des sols, une diminution des débits des cours d'eau et une réduction importante des stocks aquifères. Les conséquences de ces phénomènes ont été durement ressenties dans plusieurs secteurs de l'économie (agriculture, tourisme, navigation fluviale) et de l'écologie (eutrophisation des eaux, risque de pollution accrue, incendies de forêt,...). Selon le groupe de travail mis en place par la mission interministérielle de l'eau, la sécheresse de 1989-1990 aura marqué de façon déterminante la politique de l'eau de l'an 2000.

2. L'assèchement des sols n'a pas eu que des conséquences pour l'agriculture. Les phénomènes de retrait par dessiccation de certains terrains argileux ont provoqué de nombreux dommages aux constructions légères fondées superficiellement, par suite du tassement différentiel de leurs fondations. Ces phénomènes, d'abord observés dans le nord du pays, se sont étendus aux régions Centre, Sud-Ouest, Sud-Est et enfin lle-de-France. Parmi les départements les plus touchés, (fig. 1) citons particulièrement le Nord, le Pas-de-Calais, la Gironde, les Pyrénées-Atlantiques, l'Indre-et-Loire, la Vienne, la Seine-et-Marne, l'Indre, le Loiret, le Tarn-et-Garonne, la Charente-Maritime, le Cher, l'Essonne.

Les dommages qui en résultent sont difficiles à évaluer, eu égard à la diversité des situations et des biens affectés. Ils sont probablement considérables, compte tenu de l'extension des zones affectées et du nombre des dossiers de sinistres constitués. A titre de comparaison, l'association des assureurs britanniques (ABI) a estimé le coût des sinistres relatifs aux affaissements de sols dus à la sécheresse de 1989 , à 400 millions de livres, soit l'équivalent de 4 milliards de francs.

Le problème revêt donc un caractère grave au plan macro-économique. Il est préoccupant au regard des situations particulières de nombreux sinistrés. L'analyse d'un échantillonnage représentatif de cas de désordres, rassemblés par l'Agence Qualité Construction (1), témoigne de l'importance des dommages individuels, (évalués, par référence au coût des reprises en sousœuvre, à $100000 \mathrm{~F}$ en moyenne par unité d'habitation, avec de larges variations selon les taux d'endommagement observés et les méthodes préconisées).

3. Le dispositif prévu par la loi $n^{\circ} 82-600$ du 13 juillet 1982 relative à l'indemnisation des victimes des catastrophes naturelles (2) constitue la seule voie d'indemnisation possible, dès lors que l'état de catastrophe naturelle est reconnue et qu'un arrêté interministériel est publié au Journal Officiel. De décembre 1989 à décembre 1991, 1782 communes ont ainsi été reconnues sinistrées. Les biens directement endommagés, couverts par un contrat d'assurances « dommages » peuvent alors être indemnisés par l'assureur si la déclaration de sinistre lui est faite dans les dix jours suivant la publication de la décision administrative.
L'assureur est alors tenu de verser les indemnités prévues selon les règles du contrat de base dans un délai de 3 mois.

Les dossiers justificatifs de demande de constatation d'un état de catastrophe doivent, conformément à la circulaire interministérielle du 24 mars 1984, mettre en évidence la causalité entre "les dommages matériels directs " et "l'intensité anormale de l'agent naturel », selon les termes de l'article $1,3 e$ alinéa, de la loi du 13 juillet $1982^{*}$. Ils doivent, de ce fait, comporter des informations météorologiques qui sont rassemblées par le préfet du département mais aussi des précisions d'ordre géologique qui relèvent de l'intervention de praticiens géologues ou géotechniciens.

Les premiers dossiers soumis à la fin 1989 et au début 1990 à l'examen de la commission interministérielle chargée de leur instruction ont été étayés, à sa demande, et eu égard au caractère nouveau du problème, par des études géotechniques détaillées à la charge des personnes sinistrées. Depuis, le phénomène et ses mécanismes sont mieux connus, et les facteurs qui concourent à leur déclenchement sont identifiés. La commission interministérielle s'est donc dotée de critères d'aide à la décision, spécifiques et simples, répondant aux questions suivantes :

- l'intensité de la sécheresse peut-elle être qualifiée d'anormale?

- y-a-t'il rapport de causalité « déterminante " entre cette sécheresse et les dommages? Question amenant elle-même trois sous-questions :

- y-a-t'il corrélation temporelle entre la sécheresse observée et l'apparition de dommages?

- les sols de fondation des constructions endommagées sont-ils sensibles au phénomène de dessiccation et ont-ils été affectés par celui-ci?

- peut-on éliminer toute cause non naturelle (telle que fuite de réseaux, travaux de terrassement, remblais,...) susceptible de provoquer des effets similaires sur le bati?

4. Une réflexion globale a été engagée par le Ministère de l'environnement, avec la collaboration du Ministère de l'intérieur et de la prévention des risques technologiques et naturels majeurs, pour optimiser le contenu des dossiers et en minimiser les coûts. Dans un souci d'homogénéité avec les procédures ayant cours pour la plupart des autres événements naturels susceptibles d'être garantis, la commission n'exige plus désormais d'études géotechniques individuelles, sauf dans les cas de sinistres isolés. Dans les cas les plus fréquents, où des lotissements, des hameaux ou des quartiers entiers sont concernés, il apparaît au contraire plus opportun de procéder à des études globales à l'échelle des zones sinistrées, études basées sur une démarche d'expertise exploitant l'information existante, sans recourir à des investigations spécifiques. S'agissant d'études d'ensemble, dont les coûts n'ont plus rien à voir avec celui des études ponctuelles, les maîtrises d'ouvrage peuvent en revenir naturellement

\footnotetext{
- Sont considérés comme les effets des catastrophes naturelles, au sens de la présente loi, les dommages matériels directs ayant eu pour cause déterminante lintensité anormale d'un agent naturel lorsque les mesures habituelles à prendre pour prévenir ces dommages n'ont pu empêcher leur survenance ou n'ont pu être prises.
} 


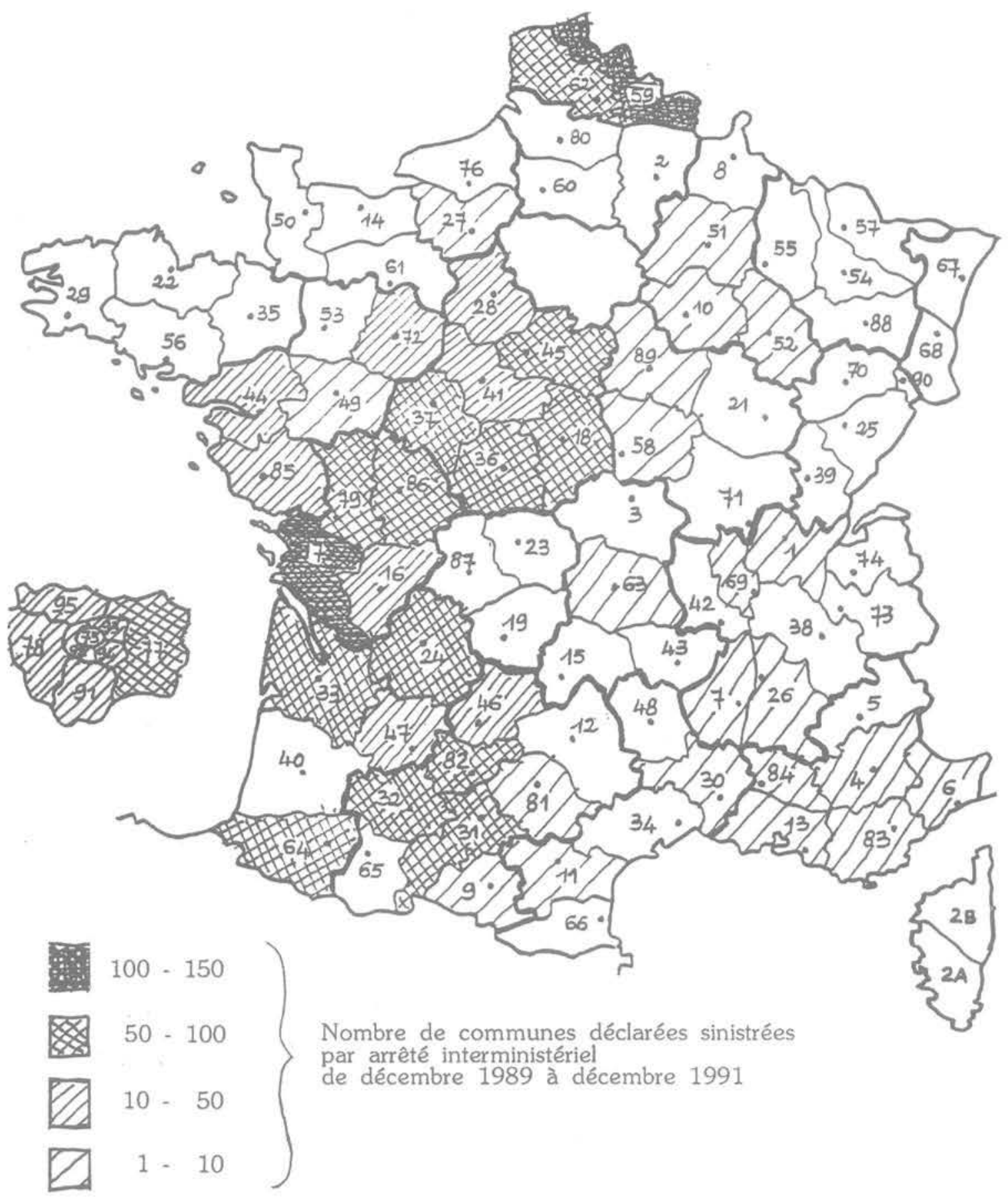

Fig. 1. - Extension des zones affectées par les dommages dus à la sécheresse, à la date du 20 mars 1991: - 1782 communes reconnues sinistrées dans 51 départements.

Fig. 1. - Accidents in France due to 1989, 1990 and 1991 droughts.

aux associations de propriétaires, aux syndicats, ou aux collectivités territoriales concernées. C'est dans cet esprit que la Direction de la Sécurité Civile a fait parvenir aux préfets de métropole des instructions parfaitement explicites pour assurer la cohérence des dossiers de demande de constatation de l'état de catastrophe naturelle. Y sont visés le contenu du dossier communal, celui du dossier de sunthèse établi par chaque préfet et la procédure de transmission à léchelon central.

Le dossier communal doit comprendre, outre un rapport circonstancié du maire, une étude géologique qualitative qualifiée de «compte rendu de visite géotechnique » et réalisée par des organismes (Bureau de 
recherche géologique et minière, Centre d'études techniques de l'équipement) ou des géotechniciens départementaux agréés. Le dossier préfectoral doit comporter une analyse météorologique témoignant de l'intensité anormale du phénomène de sécheresse. Ces dossiers sont transmis globalement au Ministère de l'Intérieur afin d'être examinés par la Commission Interministérielle dans les meilleures conditions d'efficacité et de diligence.

5. Au-delà des problèmes d'indemnisation des victimes se pose le problème du traitement du risque sous ses aspects techniques, normatifs ou réglementaires. La Délégation aux risques majeurs, en étroite collaboration avec la Direction de la sécurité civile, a engagé avec diverses instances participant à l'acte de construire et avec l'aide de praticiens compétents, une réflexion générale dans le but d'identifier les méthodes de réparation et de prévention les plus opportunes et d'en informer les personnes sinistrées ou exposées, ceci afin d'éviter le recours à des procédés inadaptés. La Délégation aux risques majeurs a été sollicitée par ailleurs par certains départements pour faire prendre en compte dans l'aménagement les risques engendrés par la dessiccation des sols sensibles. Une telle procédure implique l'intégration de ces risques dans les plans d'exposition aux risques (PER) ou les périmètres de risque délimités au titre de l'article R 111.3 du code de l'urbanisme. Des recommandations en matière d'urbanisme et de construction vont être mises au point, dans le cadre d'un groupe de travail spécialisé, afin de minimiser les conséquences d'événements météorologiques aussi graves que ceux de 1989-1991.

\section{BIBLIOGRAPHIE}

(1) LOGEAIS L. (1990), Tassement de fondations dus à la sécheresse, Sycodes informations, $\mathrm{n}^{\circ} 28$, p. $8-14$.

(2) Loi $n^{\circ} 82.600$ du 13 juillet 1982 relative à l'indemnisation des victimes de catastrophe naturelle; Journal officiel de la république française, 14 juillet 1982 , p. 2242-2243. 